\title{
Technical note: Improving modeling of coagulation, curd firming, and syneresis of sheep milk
}

\author{
Claudio Cipolat-Gotet, ${ }^{*} \dagger$ Michele Pazzola, $\ddagger^{1}$ Alessandro Ferragina, ${ }^{*}$ Alessio Cecchinato, ${ }^{*}$ Maria L. Dettori, $\ddagger$ \\ and Giuseppe M. Vaccał \\ *Department of Agronomy, Food, Natural Resources, Animals and Environment (DAFNAE), University of Padova, Viale dell'Università 16, \\ 35020 Legnaro (PD), Italy \\ †Department of Veterinary Science, University of Parma, Via del Taglio 10, 43126 Parma, Italy \\ ‡Department of Veterinary Medicine, University of Sassari, Via Vienna 2, 07100 Sassari, Italy
}

\begin{abstract}
The importance of milk coagulation properties for milk processing, cheese yield, and quality is widely recognized. The use of traditional coagulation traits presents several limitations for testing bovine milk and even more for sheep milk, due to its rapid coagulation and curd firming, and early syneresis of coagulum. The aim of this technical note is to test and improve model fitting for assessing coagulation, curd firming, and syneresis of sheep milk. Using milk samples from 87 Sarda ewes, we performed in duplicate lactodynamographic testing. On each of the 174 analyzed milk aliquots, using 180 observations from each aliquot (one every $15 \mathrm{~s}$ for $45 \mathrm{~min}$ after rennet addition), we compared 4 different curd firming models as a function of time $\left(\mathrm{CF}_{\mathrm{t}}\right.$, $\mathrm{mm}$ ) using a nonlinear procedure. The most accurate and informative results were observed using a modified 4-parameter model, structured as follows: $\mathrm{CF}_{\mathrm{t}}=\mathrm{CF}_{\mathrm{P}} \times\left(1-\mathrm{e}^{-\mathrm{k}_{\mathrm{CF}}\left(\mathrm{t}-\mathrm{RCT}_{\mathrm{eq}}\right)}\right) \times \mathrm{e}^{\mathrm{k}_{\mathrm{SR}} \times\left(\mathrm{t}-\mathrm{RCT}_{\mathrm{eq}}\right)}$, where $\mathrm{t}$ is
\end{abstract} time, $\mathrm{RCT}_{\mathrm{eq}}(\mathrm{min})$ is the gelation time, $\mathrm{CF}_{\mathrm{P}}(\mathrm{mm})$ is the potential asymptotical $\mathrm{CF}$ at an infinite time, $\mathrm{k}_{\mathrm{CF}}$ $(\% / \mathrm{min})$ is the curd firming rate constant, and $\mathrm{k}_{\mathrm{SR}}(\% /$ min) is the curd syneresis rate constant. To avoid nonconvergence and computational problems due to interrelations among the equation parameters, $\mathrm{CF}_{\mathrm{P}}$ was preliminarily defined as a function of maximum observed curd firmness $\left(\mathrm{CF}_{\max }, \mathrm{mm}\right)$ recorded during the analysis. For this model, all the modeling equations of individual sheep milk aliquots were converging, with a negligible standard error of the estimates (coefficient of determination $>0.99$ for all individual sample equations). Repeatability of the modeled parameters was acceptable, also in the presence of curd syneresis during the lactodynamographic analysis.

Received December 12, 2017.

Accepted March 8, 2018.

${ }^{1}$ Corresponding author: pazzola@uniss.it
Key words: ovine milk quality, curd firmness modeling, syneresis modeling, cheese-making, lactodynamography

\section{Technical Note}

Milk coagulation can be measured by a wide range of mechanical, vibrational, ultrasonic, thermal, and optical methods (O'Callaghan et al., 2002; Klandar et al., 2007). Among those, the monitoring with time of curd firmness (CF, mm) of renneted milk samples maintained at a fixed temperature is frequently used to assess the coagulation process (Barbano and Lynch, 2006). Although it presents several limitations, the lactodynamography analysis is extensively used to assess coagulation and curd firming process (Annibaldi et al., 1977; McMahon and Brown, 1982), as it is simple and fast, and it could be used to test contemporarily several milk samples (usually 10). In bovine milk, the major limitations of lactodynamography are related to the traditional single point traits measured by the computerized instruments and include (1) the moderate repeatability of all the traits, (2) the high incidence of noncoagulating samples (rennet coagulation time, RCT, not measurable within $30 \mathrm{~min}$ from rennet addition) and coagulated samples not attaining a curd firmness of $20 \mathrm{~mm}\left(\mathbf{k}_{\mathbf{2 0}}, \mathbf{m i n}\right)$, and (3) the low-informative value of the curd firmness traditionally measured at the end of analysis $\left(\mathbf{a}_{\mathbf{3 0}}, \mathrm{mm}\right)$. To solve those limits, Bittante (2011) presents a 3-parameter model that used all the CF measures of a sample (one every $15 \mathrm{~s}$ ) as a function of time $\left(\mathbf{C F}_{\mathbf{t}}, \mathrm{mm}\right)$ over the traditional 30-min analysis interval. The 3 estimated parameters are the RCT from the model equation $\left(\mathbf{R C T}_{\text {eq }}, \mathrm{min}\right)$, the potential asymptotic curd firmness $\left(\mathbf{C F}_{\mathbf{P}}, \mathbf{m m}\right)$, and the instant rate constant of curd firming $\left(\mathbf{k}_{\mathbf{C F}}, \% /\right.$ $\mathrm{min})$. That model reduces the limitations of lactodynamography (except for the incidence of noncoagulating samples), as the parameters are estimated using all the information (120 observations/sample) of the analysis. Moreover, the high presence of bovine milk samples 
not coagulating within 30 min suggests to extend the interval analysis to 60 or even to 90 min after rennet addition (Cipolat-Gotet et al., 2012). The extension of the analysis of bovine milk indicates $\mathrm{CF}$ reduction after the attainment of a maximum value, interpreted by Bittante et al. (2013) as a sign of the beginning of syneresis. Hence, those authors propose a new equation model introducing a fourth parameter $\left(\mathbf{k}_{\mathrm{SR}}, \% / \mathrm{min}\right)$, which describes the instant rate constant of syneresis. A limitation of those $\mathrm{CF}_{\mathrm{t}}$ models is the presence of milk samples whose equation does not converge. Nonconverging samples (i.e., very late coagulating samples), provide a few number of available observations to permit a precise estimation of modeled parameters. In those cases, the equation cannot estimate modeled parameters or outliers. Sheep milk presents a pattern of coagulation and curd firming very different from bovine milk (Bencini, 2002; Bittante et al., 2014; Ferragina et al., 2017) because the RCT is usually shorter, the curd firming more rapid, the maximum $\mathrm{CF}$ greater, and the syneresis appears earlier (often before $30 \mathrm{~min}$ after rennet addition). As a consequence, traditional coagulation traits developed on bovine milk are often not so informative for sheep milk. The aim of this technical note was to improve the $\mathrm{CF}_{\mathrm{t}}$ model fitting for assessing coagulation, curd firming, and syneresis and to test these improvements to model individual sheep milk samples.

To perform the present study, we used data from the database previously described in Pazzola et al. (2014). Among the animals sampled for that study (1,121 ewes from 23 flocks of Sarda sheep located in the island of Sardinia, Italy), 2 to 13 ewes from 18 farms were selected for a total of 87 ewes. Selected ewes were from the second to the seventh month of lactation (DIM 150 $\pm 47 \mathrm{~d})$ and had a daily milk yield of $1.53 \pm 0.78$ $\mathrm{kg} / \mathrm{d}$. After the collection, milk samples were divided into 2 aliquots $\left(50 \mathrm{~mL}\right.$ each) and kept at $4^{\circ} \mathrm{C}$ (without preservative). Traditional single-point traits (RCT, $\mathrm{k}_{20}, \mathrm{a}_{30}, \mathrm{a}_{45}$, and $\mathrm{a}_{60}$ ) and all the CF measures (240 for each milk aliquot, 1 every $15 \mathrm{~s}$ ) were measured using a lactodynamograph (Formagraph, Foss Italia, Padova, Italy) and prolonging the test length until $60 \mathrm{~min}$. Two aliquots of each individual milk sample $(10 \mathrm{~mL}$ each aliquot) were heated at $35^{\circ} \mathrm{C}$ and mixed with $200 \mu \mathrm{L}$ of the calf rennet solution [Hansen Naturen Plus 215 (Pacovis Amrein AG, Bern, Switzerland), with $80 \pm$ $5 \%$ chymosin and $20 \pm 5 \%$ pepsin and 215 international milk clotting units $/ \mathrm{mL}$; diluted to $1.2 \%$ (wt/ vol) in distilled water for the achievement of 0.0513 international milk clotting units/mL of milk]. For all the milk aliquots $(\mathrm{n}=174)$, RCT and $\mathrm{k}_{20}$ were achievable within the first $45 \mathrm{~min}$ of the lactodynamographic analysis. Therefore, to compare different $\mathrm{CF}_{\mathrm{t}}$ models, only the first $45 \mathrm{~min}$ (180 observations per aliquot) were used to estimate model parameters, as this is considered an adequate time interval to attain syneresis in individual ewe milk samples (Vacca et al., 2015). We tested 4 different nonlinear models for curd firming $\left(\mathrm{CF}_{\mathrm{t}}, \mathrm{mm}\right)$. The first model $\left(\mathbf{3 P a r}_{\text {fixed }}\right)$ was the 3 -parameter asymptotic model proposed by Bittante (2011):

$$
\mathrm{CF}_{\mathrm{t}}=\mathrm{CF}_{\mathrm{P}} \times\left(1-\mathrm{e}^{-\mathrm{k}_{\mathrm{CF}}\left(\mathrm{t}-\mathrm{RCT}_{\mathrm{eq}}\right)}\right)
$$

where $\mathrm{CF}_{\mathrm{t}}$ is $\mathrm{CF}$ at time $\mathrm{t}(\mathrm{mm}) ; \mathrm{CF}_{\mathrm{P}}$ is the asymptotical potential value of $\mathrm{CF}$ at an infinite time (mm); $\mathrm{k}_{\mathrm{CF}}$ is the curd firming rate constant $(\% / \mathrm{min})$ and describes the shape of the curve from coagulation time to infinity (velocity of curd firming); and $\mathrm{RCT}_{\mathrm{eq}}$ has the same meaning as the traditional RCT trait. A second model $\left(\mathbf{3 P a r}_{\text {variable }}\right)$ had the same equation as above but was based on a different and variable number of observations $(\leq 180)$. In this second model, the observed maximum $\mathrm{CF}$ value $\left(\mathbf{C F}_{\max }, \mathbf{m m}\right)$ for each analyzed aliquot was used to measure the corresponding time after rennet addition $\left(\mathbf{t}_{\max }, \mathrm{min}, \leq 45 \mathrm{~min}\right)$. Only the $\mathrm{CF}$ observations between the rennet addition and $t_{\max }$ were used for the estimation of the parameters of $3 \mathrm{Par}_{\text {variable }}$ model. In the case of aliquots presenting tendency to asymptote, the first value of $\mathrm{CF}_{\max }$ was used if more than one maximum value was recorded during the single analysis. In this way, the potential decreasing phase of the $\mathrm{CF}_{\mathrm{t}}$ curve was excluded from calculations, the number of observations was retained, and the corresponding degrees of freedom were different among the analyzed aliquots. The third was a 4-parameter model $\left(4 \mathrm{Par}_{\text {free }}\right)$ previously proposed for bovine milk by Bittante et al. (2013), characterized by prolonging the duration of the lactodynamographic test and including the aforementioned parameters together with $\mathrm{k}_{\mathrm{SR}}(\% /$ min), the instant rate constant of syneresis, as follows:

$$
\mathrm{CF}_{\mathrm{t}}=\mathrm{CF}_{\mathrm{P}} \times\left(1-\mathrm{e}^{-\mathrm{k}_{\mathrm{CF}}\left(\mathrm{t}-\mathrm{RCT}_{\mathrm{eq}}\right)}\right) \times \mathrm{e}^{\mathrm{k}_{\mathrm{SR}} \times\left(\mathrm{t}-\mathrm{RCT}_{\mathrm{eq}}\right)} .
$$

The $4 \mathrm{Par}_{\text {free }}$ model was proposed as a large percentage of individual milk samples showed a decreasing phase of $\mathrm{CF}_{\mathrm{t}}$, sometimes within $30 \mathrm{~min}$ after rennet addition (Vacca et al., 2015). In the first part of the curve, the effect of $\mathrm{k}_{\mathrm{CF}}$ prevails over $\mathrm{k}_{\mathrm{SR}}$, and $\mathrm{CF}_{\mathrm{t}}$ tends to increase until $t_{\max }$. At $t_{\max }$ the effects of $k_{\mathrm{CF}}$ and $\mathrm{k}_{\mathrm{SR}}$ are equal and opposite in sign. At $t_{\max }$, the effects of these 2 traits are equal and opposite. After $t_{\max }$, the effect of syneresis becomes more forceful than $\mathrm{k}_{\mathrm{CF}}$, and $\mathrm{CF}_{\mathrm{t}}$ curve tends to decrease toward a null value. The decrease of $\mathrm{CF}$ is apparent and is due to the increasing 
amount of whey in the cuvette that allows the curd to float freely so that the pendulum of the instrument finds a decreased resistance to movement. As the rate constant $\mathrm{k}_{\mathrm{SR}}$ is associated with this part of the curve (from $t_{\max }$ to the end of testing), it describes the effect of the corresponding expulsion of whey. The $4 \operatorname{Par}_{\text {free }}$ model estimates all the 4 parameters directly from all the $180 \mathrm{CF}$ available measures. The $\mathbf{4} \mathbf{P a r}_{\text {forced }}$ model was derived from $4 \mathrm{Par}_{\text {free }}$ and was proposed to limit convergence and estimation problems: in this case, $\mathrm{CF}_{\mathrm{P}}$ was calculated by multiplying $\mathrm{CF}_{\max }$ with 1.12 . This multiplicative coefficient was obtained from the linear regression between $\mathrm{CF}_{\mathrm{P}}$ and $\mathrm{CF}_{\max }$ values, estimated in a preliminary analysis of the entire data set using $4 \operatorname{Par}_{\text {free }}$ (Vacca et al., 2015). Then, using the $4 \mathrm{Par}_{\text {forced }}$ modeling procedure, we estimated directly only 3 parameters $\left(\mathrm{RCT}_{\mathrm{eq}}, \mathrm{k}_{\mathrm{CF}}\right.$, and $\left.\mathrm{k}_{\mathrm{SR}}\right)$ using all $180 \mathrm{CF}$ observations, whereas $\mathrm{CF}_{\mathrm{P}}$ was preliminarily estimated as a function of the observed $\mathrm{CF}_{\max }$.

For the parameters estimation of the 4 models $\left(3 \mathrm{Par}_{\text {fixed }}, 3 \mathrm{Par}_{\text {variable }}, 4 \mathrm{Par}_{\text {free }}\right.$, and $\left.4 \mathrm{Par}_{\text {forced }}\right)$, we used univariate curvilinear regressions based on all the $\mathrm{CF}$ observations available for each aliquot with a nonlinear procedure (PROC NLIN; SAS version 9.4, SAS Institute Inc., Cary, NC). The parameters of each individual equation were estimated by using the Marquardt iterative method (350 iterations and $10^{-5}$ level of convergence). To test the fitting of the models, individual modeled $\mathrm{CF}_{\mathrm{t}}$ traits (2 replicates per ewe), together with traditional traits, were analyzed using a MIXED procedure (SAS Institute Inc.) that included the fixed effect of well (measuring unit of the coagulation meter) and the random effects of herd/date, animal, and residual (called aliquot). Herd/date, animal, and aliquot were assumed to be independently and normally distributed with a mean of zero and variance $\sigma_{H D}^{2}, \sigma_{\text {animal }}^{2}$, and $\sigma_{\text {aliquot }}^{2}$, respectively. The coefficient of repeatability (REP) was estimated as

$$
\mathrm{REP}=\frac{\sigma_{H D}^{2}+\sigma_{\text {animal }}^{2}}{\sigma_{H D}^{2}+\sigma_{\text {animal }}^{2}+\sigma_{\text {aliquot }}^{2}} \times 100,
$$

where $\sigma_{H D}^{2}, \sigma_{\text {animal }}^{2}$, and $\sigma_{\text {aliquot }}^{2}$ are the variance components for herd/date, animal, and milk sub-sample from the same individual ewe, respectively.

As shown by the descriptive statistics (Table 1), results of traditional and modeled milk coagulation properties are similar or more favorable than previous studies on Sarda sheep milk (Pazzola et al., 2014; Vacca et al., 2015; Manca et al., 2016). Figure 1 presents an example of how models can fit the pattern of lactodynamographic analyses of an individual aliquot of ovine milk. The performance of the models was evaluated on the basis of the percentage of individual milk sample aliquots with convergent equations and by estimating the coefficient of determination of the convergent individual equations. We observed the best results for $3 \mathrm{Par}_{\text {variable }}$, $4 \mathrm{Par}_{\text {free }}$, and $4 \mathrm{Par}_{\text {forced }}$. These model were characterized by coefficients of determination higher than 0.99 and negligible residual standard deviations (Figure 2). In opposite, $3 \mathrm{Par}_{\text {fixed }}$ showed the lowest coefficients of determination and the highest residual standard deviations (Figure 2); moreover, 3 individual aliquot equations were not convergent. Although 4 Par $_{\text {free }}$ exhibited good fitting results, 6 out of the 174 aliquots were nonconvergent (data not shown). When a milk sample exhibits a nonconvergent equation, all the predicted modeled traits have to be considered missing. Nonconverging $\mathrm{CF}_{\mathrm{t}}$ model equations are more common for late-coagulating samples (long RCT) or with very high $\mathrm{k}_{\mathrm{CF}}$ and $\mathrm{CF}_{\max }$, respectively, and for those samples characterized by absence or nonlinear trend in the syneresis process. Using $4 \mathrm{Par}_{\text {free }}$, nonconvergent individual milk samples are $7 \%$ for bovine (Bittante et al., 2015) and almost 5\% for sheep milk (Vacca et al., 2015).

In the present study, models with only 3 parameters were not able to explain the appropriate pattern of milk samples characterized by a decreasing phase of $\mathrm{CF}_{\mathrm{t}}$ curve due to curd syneresis and whey expulsion (Figure 1). Figure 1 also showed that the exclusion of the decreasing part of the curve $\left(3 \mathrm{Par}_{\text {variable }}\right)$ allowed to obtain a more reliable estimation of $\mathrm{CF}_{\mathrm{P}}$ and $\mathrm{k}_{\mathrm{CF}}$ parameters and to fit the curve until $\mathrm{CF}_{\max }$. Among parameters of the models, $\mathrm{RCT}_{\mathrm{eq}}$ estimates were similar for all models and slightly greater, on average, than the traditional RCT (Table 1). The curvilinear graph shape before and after RCT (no singular point, or cusp, is detectable) for observed data (Figure 1) derived from the lactodynamograph mechanism, which mitigates the curve line by taking a mobile average of $\mathrm{CF}_{\mathrm{t}}$ data. Therefore, measured RCT is recorded when $\mathrm{CF}_{t}$ is lower than $1 \mathrm{~mm}$, whereas $\mathrm{RCT}_{\text {eq }}$ is estimated when $\mathrm{CF}_{\mathrm{t}}$ is $0 \mathrm{~mm}$. The $3 \mathrm{Par}_{\text {fixed }}$ model underestimated $\mathrm{CF}_{\mathrm{P}}$ because of the bias induced by the decreasing phase of $\mathrm{CF}_{\mathrm{t}}$ curve, which was excluded from computations of parameters from $3 \mathrm{Par}_{\text {variable }}$. The $3 \mathrm{Par}_{\text {variable }}$ model was particularly appropriate when the decreasing phase was not evident in the $\mathrm{CF}_{\mathrm{t}}$ curve, as in the case of sheep milk samples tested for short periods, or in the case of late-coagulating bovine milk samples, as frequently observed in milk from Holstein Friesian cows (Bittante et al., 2012).

Figure 1 shows that the models including $\mathrm{k}_{\mathrm{SR}}\left(4 \operatorname{Par}_{\text {free }}\right.$ and $\left.4 \mathrm{Par}_{\text {forced }}\right)$ were able to describe the curves presenting decreasing phases. The differences between observed and predicted $\mathrm{CF}$ measurements for these 2 models can 
be defined as negligible because of the moderate repeatability and reproducibility that characterizes the lactodynamographic analysis (Stocco et al., 2015). However, regardless of the fitting of the 4Par models, the limitation of $\mathrm{k}_{\mathrm{SR}}$ is mainly attributable to the descending phase of the curve, which reflects the expulsion of the whey (and not a direct measure of $\mathrm{CF}$ ), and it has to be considered only an indirect measure of syneresis. The two 4Par models presented higher $\mathrm{CF}_{\mathrm{P}}$ estimates than 3-parameter models because the effect of syneresis caused a partial disguising of the actual CF. This is in accordance with data recorded from bovine milk (Bittante et al., 2013). As expected, the $\mathrm{k}_{\mathrm{CF}}$ rate constant of both the 4-parameter models was smaller than those of the 3-parameter models because of the greater $\mathrm{CF}_{\mathrm{P}}$. Results of ANOVA (Table 1) were used to evaluate the reproducibility among different wells, sampling date/ herds, individual ewes, and the repeatability of modeled traits measured between the 2 aliquots of each milk sample. The $\mathrm{RCT}$, and $\mathrm{k}_{\mathrm{CF}}$ and $\mathrm{k}_{\mathrm{SR}}$ from the $4 \mathrm{Par}_{\text {free }}$ model, were not affected by the well effect. Contrary to traditional $\mathrm{RCT}, \mathrm{RCT}_{\text {eq }}$ was significantly affected by well. This was not attributable to the effect of the wells on this trait, but to its much lower residual (aliquot) variance. Indeed, the aliquot RMS is about halved in modeled than in single point RCT, so that its variance is about one-fourth and then the F-value of the well is about 4 times higher for $\mathrm{RCT}_{\text {eq }}$ (Table 1 ). Also for the 2

Table 1. Descriptive statistics, ANOVA, and repeatability of traditional single point milk coagulation properties (MCP) and curd firming models as a function of time $\left(\mathrm{CF}_{\mathrm{t}}\right)$ model parameters estimated for each milk sample aliquot according to $3 \mathrm{Par}_{\text {fixed }}, 3 \mathrm{Par}_{\text {variable }}, 4 \mathrm{Par}$ free, and $4 \mathrm{Par}_{\text {forced }}$ models $^{1}$

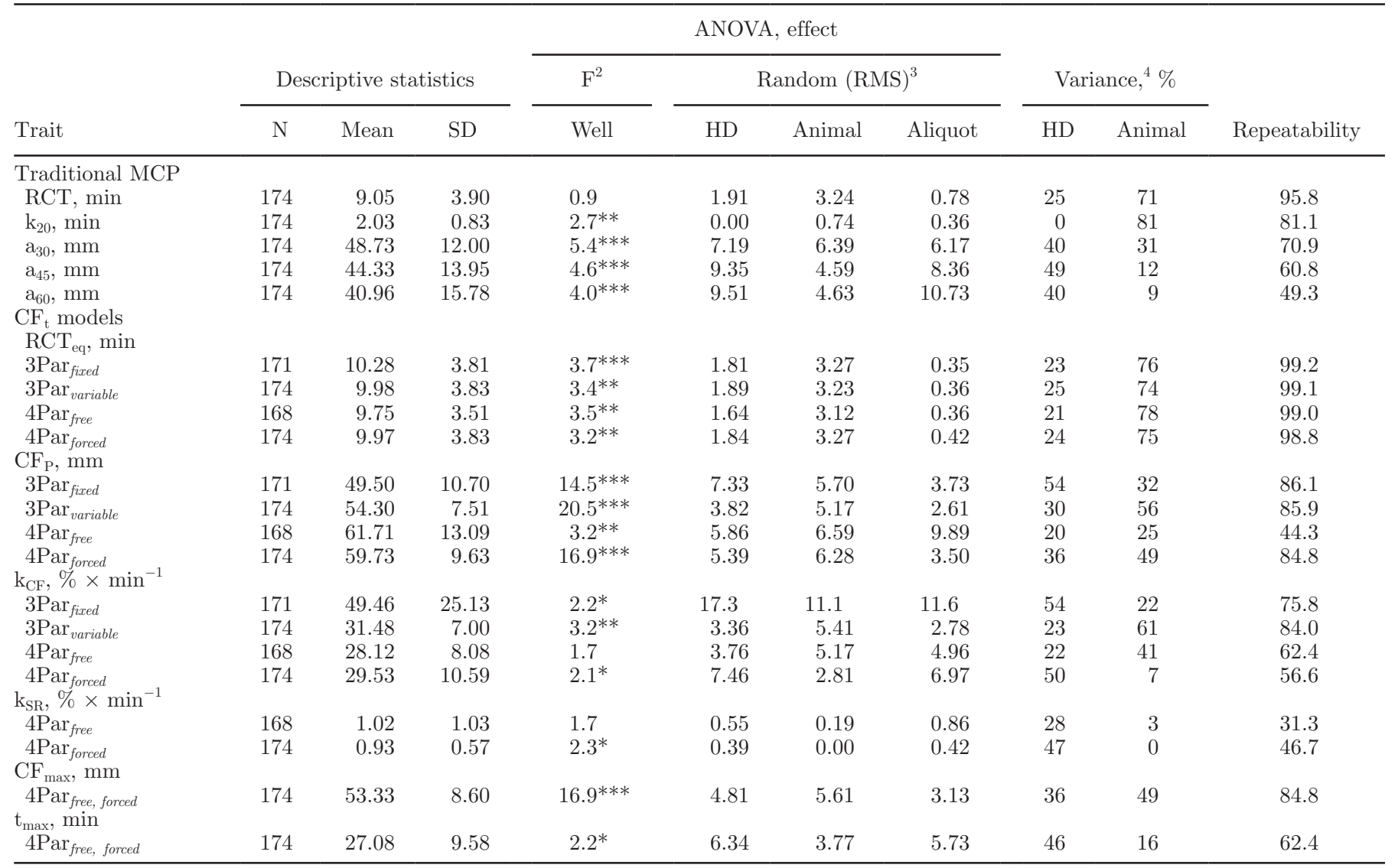

${ }^{1} \mathrm{RCT}=$ rennet coagulation time; $\mathrm{k}_{20}=$ coagulated samples not attaining a curd firmness of $20 \mathrm{~mm} ; \mathrm{a}_{30}=$ curd firmness traditionally measured at the end of analysis; $\mathrm{a}_{45}=; \mathrm{a}_{60}=; \mathrm{CF}_{\mathrm{P}}=$ potential asymptotical $\mathrm{CF}$ at an infinite time; $\mathrm{k}_{\mathrm{CF}}=$ the curd firming rate constant; $\mathrm{CF}_{\max }=$ maximum observed curd firmness; and $\mathrm{t}_{\max }=$ maximum time after rennet addition. $3 \mathrm{Par}_{\text {fixed }}=3$-parameter asymptotic model proposed by Bittante (2011); $3 \mathrm{Par}_{\text {variable }}=$ same equation as $3 \mathrm{Par}_{\text {fixed }}$ but based on a different and variable number of observations $(\leq 180)$; 4 Par free $=4$-parameter model previously proposed for bovine milk by Bittante et al. (2013); $4 \mathrm{Par}_{\text {forced }}=$ model derived from $4 \mathrm{Par}_{\text {free }}$ and was proposed to limit convergence and estimation problems; $4 \mathrm{Par}_{\text {free, forced }}=\mathrm{CF}_{\max }$ and $\mathrm{t}_{\max }$ were estimated using the same procedures for both models.

${ }^{2} \mathrm{~F}=\mathrm{F}$-value of fixed effect of well.

${ }^{3} \mathrm{RMS}=$ root mean squares of random effects [herd day (HD), animal, and aliquot, respectively].

${ }^{4} \mathrm{HD}$ and animal variance are expressed by the ratio between the corresponding variance component and the total variance (\%).

${ }^{*} P<0.05 ;{ }^{* *} P<0.01 ;{ }^{* * *} P<0.001$. 


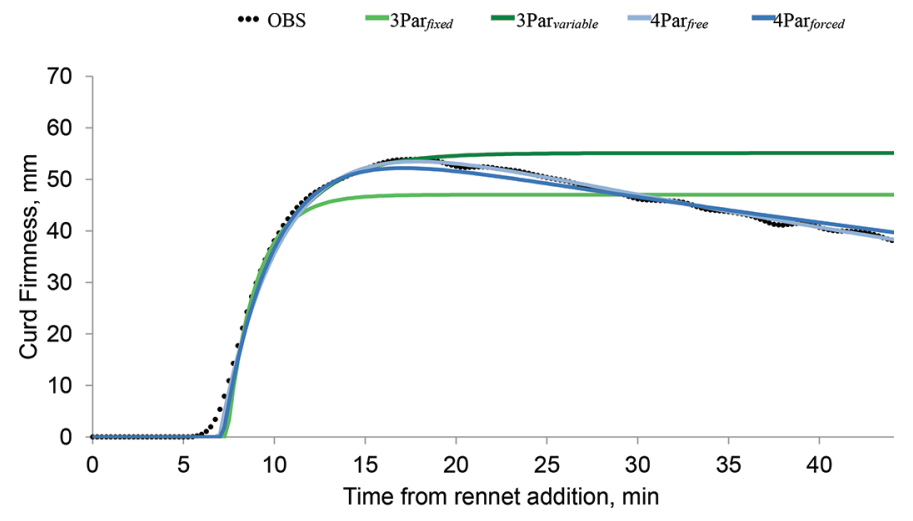

Figure 1. Modeled pattern of curd firmness after rennet addition [curd firming models as a function of time $\left(\mathrm{CF}_{\mathrm{t}}\right)$ modeling] of an individual sheep milk sample using $3 \mathrm{Par}_{\text {fixed }}, 3 \mathrm{Par}_{\text {variable }}, 4 \mathrm{Par}_{\text {free }}$, and $4 \mathrm{Par}_{\text {forced }}$ models. OBS $=$ observed; $3 \mathrm{Par}_{\text {fixed }}=3$-parameter asymptotic model proposed by Bittante (2011); $3 \mathrm{Par}_{\text {variable }}=$ same equation as $3 \mathrm{Par}_{\text {fixed }}$ but based on a different and variable number of observations $(\leq 180) ; 4 \mathrm{Par}_{\text {free }}=4$-parameter model previously proposed for bovine milk by Bittante et al. (2013); $4 \mathrm{Par}_{\text {forced }}=$ model derived from $4 \mathrm{Par}_{\text {free }}$ and was proposed to limit convergence and estimation problems. Color version available online.

constant rates obtained from $4 \mathrm{Par}_{\text {free }}$ model, the aliquot root means square was relatively high. These results were mostly related to the decrease of accuracy and repeatability (Table 1) that characterized CF measures from RCT to the end of lactodynamographic analyses. The effect of wells was particularly evident for the $\mathrm{CF}_{\mathrm{P}}$ and $\mathrm{CF}_{\max }$ traits, confirming the results obtained from bovine milk (Stocco et al., 2017). With regard to random effects, we observed a higher repeatability (REP) for RCT than the other traits. The results in terms of REP among the 4 models were different among modeled parameters. In the case of $\mathrm{RCT}_{\mathrm{eq}}$, all the 4 models showed excellent REP values, higher than the traditional RCT (Table 1). Comparing the 3-parameter models, REP was similar for $\mathrm{CF}_{\mathrm{P}}$ and greater for $3 \mathrm{Par}_{\text {variable }}$ than $3 \mathrm{Par}_{\text {fixed }}$ in the case of $\mathrm{k}_{\mathrm{CF}}$. Among parameters obtained using $4 \mathrm{Par}_{\text {free }}$, the REP value for $\mathrm{CF}_{\mathrm{P}}$ and $\mathrm{k}_{\mathrm{CF}}$ was lower compared with the 3-parameter models, and very low for $\mathrm{k}_{\mathrm{SR}}$ rate. This was attributable to the interrelations among the 4 parameters. The adoption of a preliminary estimate of $\mathrm{CF}_{\mathrm{P}}$ on the basis of $\mathrm{CF}_{\max }\left(4 \mathrm{Par}_{\text {forced }}\right.$ model) reduced the interdependency of parameters and allowed improvement of the REP of both $\mathrm{CF}_{\mathrm{P}}$ and $\mathrm{k}_{\mathrm{SR}}$ (Table 1).

In conclusion, the $3 \mathrm{Par}_{\text {variable }}$ model could be a valid tool for representing coagulation and curd-firming processes when the length of the lactodynamographic test was limited, when milk coagulation was delayed, when the $\mathrm{CF}_{\mathrm{t}}$ curve did not present a decreasing phase, or even when the eventual decreasing or flat section of the curve was excluded from calculations. The 4-parameter models offered a better fitting of $\mathrm{CF}_{\mathrm{t}}$ curves in all the other cases and new indirect information regarding syneresis. In this case, the preliminary definition of $\mathrm{CF}_{\mathrm{P}}$ on the basis of $\mathrm{CF}_{\max }$ reduced the interdependency of modeled parameters, allowed more repeatable and affordable parameters, and the convergence of the individual milk samples equation $\left(4 \mathrm{Par}_{\text {forced }}\right.$ model). Although practi-

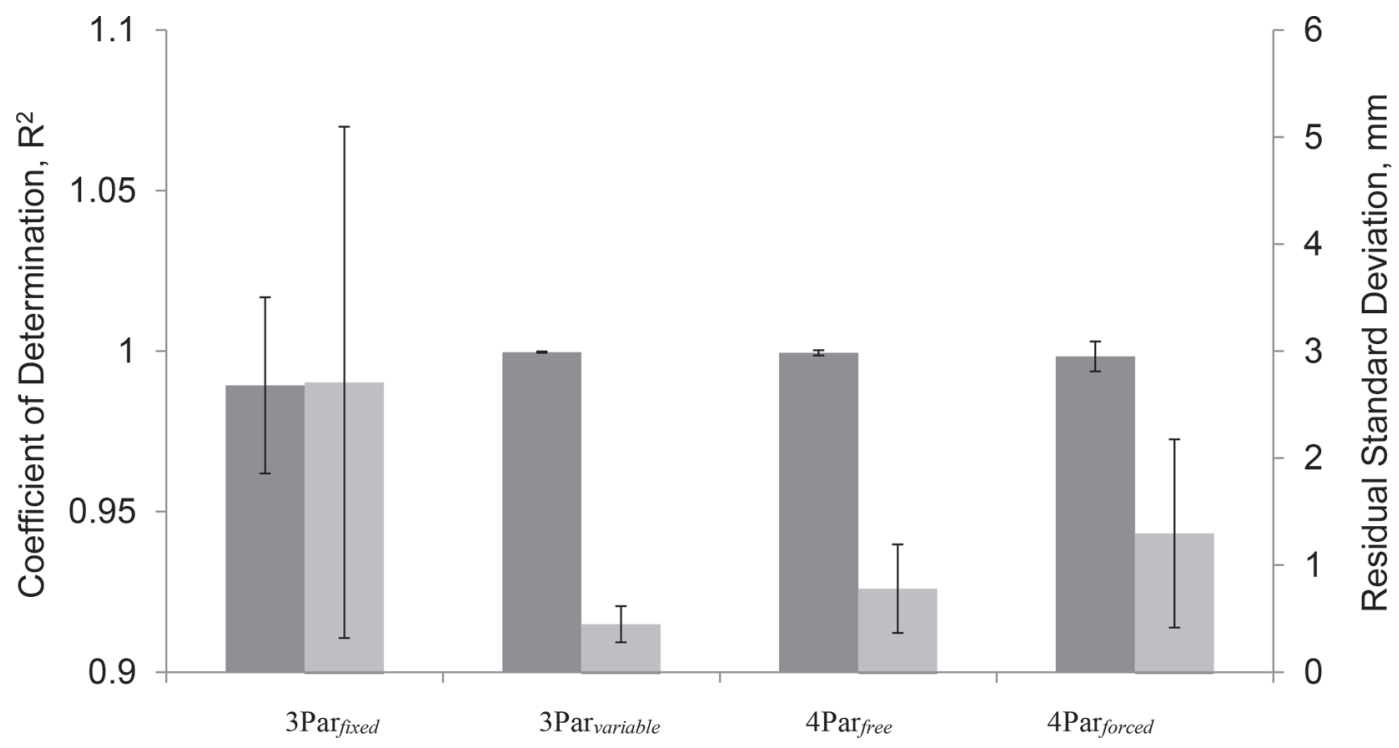

Figure 2. Coefficients of determination (dark bars) and residual SD (light bars) for model fitting results obtained for $3 \mathrm{Par}_{\text {fixed }}, 3 \mathrm{Par}$ variable $4 \mathrm{Par}_{\text {free }}$, and $4 \mathrm{Par}_{\text {forced }}$ models. $3 \mathrm{Par}_{\text {fixed }}=3$-parameter asymptotic model proposed by Bittante (2011); $3 \mathrm{Par}_{\text {variable }}={\text { same equation as } 3 \mathrm{Par}_{\text {fixed }} \text { but }}$ based on a different and variable number of observations $(\leq 180) ; 4 \mathrm{Par}_{\text {free }}=4$-parameter model previously proposed for bovine milk by Bittante et al. (2013); $4 \mathrm{Par}_{\text {forced }}=$ model derived from $4 \mathrm{Par}_{\text {free }}$ and was proposed to limit convergence and estimation problems. 
cal application of proposed methods for inline use still has to be evaluated, this new knowledge can be useful to promote lactodynamographic tests along the dairy chain to better characterize individual milk samples at farm level and to improve milk payment systems.

\section{ACKNOWLEDGMENTS}

This research was supported by a grant from the Fondazione di Sardegna (Sardinia Foundation, Italy). The authors thank the farmers for giving access to their farms, the AIPA/APA (Provincial Farmers Associations) of Cagliari, Nuoro, Sassari, and Oristano for their support in sample collection, and ARA Sardegna (Regional Farmer Association of Sardinia) for support in milk analysis. The authors gratefully thank Giovanni Bittante (Department of Agronomy, Food, Natural Resources, Animals and Environment, University of Padova, Italy) for his valuable comments and suggestions to improve the quality of the manuscript.

\section{REFERENCES}

Annibaldi, S., F. Ferri, and R. Morra. 1977. Nuovi orientamenti nella valutazione tecnica del latte: Tipizzazione lattodinamografica. Sci. Tecn. Latt. Cas. 28:115-126.

Barbano, D. M., and J. M. Lynch. 2006. Major advances in testing of dairy products: Milk component and dairy product attribute testing. J. Dairy Sci. 89:1189-1194.

Bencini, R. 2002. Factors affecting the clotting properties of sheep milk. J. Sci. Food Agric. 82:705-719.

Bittante, G. 2011. Modeling rennet coagulation time and curd firmness of milk. J. Dairy Sci. 94:5821-5832.

Bittante, G., C. Cipolat-Gotet, F. Malchiodi, E. Sturaro, F. Tagliapietra, S. Schiavon, and A. Cecchinato. 2015. Effect of dairy farming system, herd, season, parity and days in milk on modeling of coagulation, curd firming and syneresis of bovine milk. J. Dairy Sci. 98:2759-2774.

Bittante, G., B. Contiero, and A. Cecchinato. 2013. Prolonged observation and modelling of milk coagulation, curd firming, and syneresis. Int. Dairy J. 29:115-123.
Bittante, G., E. Pellattiero, F. Malchiodi, C. Cipolat-Gotet, M. Pazzola, G. M. Vacca, S. Schiavon, and A. Cecchinato. 2014. Quality traits and modelling of coagulation, curd firming and syneresis of sheep milk of Alpine breeds fed diets supplemented with rumen protected conjugated fatty acid. J. Dairy Sci. 97:4018-4028.

Bittante, G., M. Penasa, and A. Cecchinato. 2012. Invited review: Genetics and modeling of milk coagulation properties. J. Dairy Sci. 95:6843-6870.

Cipolat-Gotet, C., A. Cecchinato, M. De Marchi, M. Penasa, and G. Bittante. 2012. Comparison between mechanical and near-infrared methods for assessing coagulation properties of bovine milk. J. Dairy Sci. 95:6806-6819.

Ferragina, A., C. Cipolat-Gotet, A. Cecchinato, M. Pazzola, M. L. Dettori, G. M. Vacca, and G. Bittante. 2017. Prediction and repeatability of milk coagulation properties and curd-firming modeling parameters of ovine milk using Fourier-transform infrared spectroscopy and Bayesian models. J. Dairy Sci. 100:3526-3538.

Klandar, A. H., A. Lagaude, and D. Chevalier-Lucia. 2007. Assessment of the rennet coagulation of skim milk: A comparison of methods. Int. Dairy J. 17:1151-1160.

Manca, M. G., J. Serdino, G. Gaspa, P. Urgeghe, I. Ibba, M. Contu, P. Fresi, and N. P. P. Macciotta. 2016. Derivation of multivariate indices of milk composition, coagulation properties, and individual cheese yield in dairy sheep. J. Dairy Sci. 99:4547-4557.

McMahon, D. J., and R. J. Brown. 1982. Evaluation of Formagraph for comparing rennet solutions. J. Dairy Sci. 65:1639-1642.

O'Callaghan, D. J., C. P. O'Donnell, and F. A. Payne. 2002. Review of systems for monitoring curd setting during cheesemaking. Int. J. Dairy Technol. 55:65-67.

Pazzola, M., M. L. Dettori, C. Cipolat-Gotet, A. Cecchinato, G. Bittante, and G. M. Vacca. 2014. Phenotypic factors affecting coagulation properties of milk from Sarda ewes. J. Dairy Sci. 97:72477257.

Stocco, G., C. Cipolat-Gotet, T. Bobbo, A. Cecchinato, and G. Bittante. 2017. Breed of cow and herd productivity affect milk composition and modeling of coagulation, curd firming, and syneresis. J. Dairy Sci. 100:129-145.

Stocco, G., C. Cipolat-Gotet, A. Cecchinato, L. Calamari, and G. Bittante. 2015. Milk skimming, temperature, acidification, lysozyme, and rennet affect the pattern, repeatability and predictability of milk coagulation properties and of curd firming model parameters: the case-study of Grana Padano. J. Dairy Sci. 98:5052-5067.

Vacca, G. M., M. Pazzola, M. L. Dettori, E. Pira, F. Malchiodi, C. Cipolat-Gotet, A. Cecchinato, and G. Bittante. 2015. Modeling of coagulation, curd firming, and syneresis of milk from Sarda ewes. J. Dairy Sci. 98:2245-2259. 